\begin{tabular}{|c|l|}
\hline Title & $\begin{array}{l}\text { Canonical commutation relations, the W eierstrass Zeta function, and infinite dimensional Hil bert space representations } \\
\text { of the quantum group U q(s/2) }\end{array}$ \\
\hline Author(s) & A rai, A sao \\
\hline Citation & $\begin{array}{l}\text { Journal of Mathematical Physics, 37(9), 4203-4218 } \\
\text { https://doi.org/40.1063/1.531797 }\end{array}$ \\
\hline Issue Date & 1996-09 \\
\hline Doc URL & http://hdl.handle.net/2115/13672 \\
\hline Rights & Copyright $\odot 1996$ A merican Institute of Physics \\
\hline Type & article \\
\hline File Information & jmp37-9.pdf \\
\hline
\end{tabular}

Instructions for use 


\title{
Canonical commutation relations, the Weierstrass Zeta function, and infinite dimensional Hilbert space representations of the quantum group $U_{q}\left(\mathfrak{s l}_{2}\right)$
}

\author{
Asao Arai ${ }^{\mathrm{a})}$ \\ Department of Mathematics, Hokkaido University, Sapporo 060, Japan
}

(Received 13 November 1995; accepted for publication 25 April 1996)

\begin{abstract}
A two-dimensional quantum system of a charged particle interacting with a vector potential determined by the Weierstrass Zeta function is considered. The position and the physical momentum operators give a representation of the canonical commutation relations with two degrees of freedom. If the charge of the particle is not an integer (the case corresponding to the Aharonov-Bohm effect), then the representation is inequivalent to the Schrödinger representation. It is shown that the inequivalent representation induces infinite-dimensional Hilbert space representations of the quantum group $U_{q}\left(\mathfrak{s l}_{2}\right)$. Some properties of these representations of $U_{q}\left(\mathfrak{s l}_{2}\right)$ are investigated. (C) 1996 American Institute of Physics.
\end{abstract}

[S0022-2488(96)00108-9]

\section{INTRODUCTION}

In a previous paper, ${ }^{1}$ we considered a quantum system of a charged particle moving in the Euclidean plane $\mathbf{R}^{2}$ under the influence of a perpendicular magnetic field, which may be strongly singular at some fixed points $\mathbf{a}_{1}, \ldots, \mathbf{a}_{N}$ in $\mathbf{R}^{2}$. If the magnetic field is concentrated on the discrete set $\left\{\mathbf{a}_{n}\right\}_{n=1}^{N}$ in the sense of distribution, then the position and the physical momentum operators give a representation of the canonical commutation relations (CCRs) (Heisenberg relations) with two degrees of freedom.

Here we recall some technical terms in the representation theory of CCR. A set $\left\{\mathscr{H}, \mathscr{D},\left\{Q_{j}, P_{j}\right\}_{j=1}^{d}\right\}$ consisting of a Hilbert space $\mathscr{H}$, a dense subspace $\mathscr{D}$ of $\mathscr{H}$, and self-adjoint operators $Q_{j}, P_{j},(j=1, \ldots, d)$ is called a representation of the CCR with $d$ degrees of freedom if $\mathscr{D} \subset \cap_{j, k=1}^{d}\left[D\left(Q_{j} Q_{k}\right) \cap D\left(Q_{j} P_{k}\right) \cap D\left(P_{k} Q_{j}\right) \cap D\left(P_{j} P_{k}\right)\right][D(T)$ denotes the domain of operator $T]$ and the CCR,

$$
\left.\left.\left[Q_{j}, Q_{k}\right]=0,\right\urcorner\left[P_{j}, P_{k}\right]=0, \neg\left[Q_{j}, P_{k}\right]=i \delta_{j k},\right\urcorner j, k=1, \ldots, d,
$$

hold on $\mathscr{D}$, where $[S, T]:=S T-T S$. Following Putnam, ${ }^{2}$ we say that a set $\left\{Q_{j}, P_{j}\right\}_{j=1}^{d}$ of selfadjoint operators on a Hilbert space is a Schrödinger $d$ system if it is unitarily equivalent to a direct sum of the Schrödinger representation of the CCR with $d$ degrees of freedom. A representation $\left\{\mathscr{H}, \mathscr{D},\left\{Q_{j}, P_{j}\right\}_{j=1}^{d}\right\}$ of CCR is called equivalent (resp., inequivalent) if $\left\{Q_{j}, P_{j}\right\}_{j=1}^{d}$ is (resp., not) a Schrödinger $d$ system.

In Ref. 1 the following facts were shown: (i) the representation of CCR mentioned above is equivalent if and only if the magnetic flux is locally quantized (i.e., the magnetic flux at each point $\mathbf{a}_{n}(n=1, \ldots, N)$ is an integer multiple of $2 \pi / \alpha$, where $\alpha$ is the charge of the particle); (ii) the inequivalent representation appearing in the case where the magnetic flux is not locally quantized may be regarded as a mathematical form of the Aharonov-Bohm effect. ${ }^{3}$ A geometric construction of a representation of CCR that is unitarily equivalent to the one given in Ref. 1 was considered by Kurose and Nakazato, ${ }^{4}$ and more detailed properties of the representation were discussed.

${ }^{a)}$ Electronic mail: irai@math.hokudai.ac.jp 
To investigate differences between the equivalent and the inequivalent representations of CCR in Ref. 1, an analysis has been made on Dirac-Weyl operators defined in terms of the physical momentum operator. ${ }^{5}$ Moreover, the framework and results in Ref. 1 were extended to the case of a non-Abelian gauge theory. 6,7

Recently Wiegmann and Zabrodin ${ }^{8}$ considered a quantum system of a particle on a twodimensional square lattice in a magnetic field and showed that magnetic translations on the lattice are related to finite-dimensional representations of the quantum group $U_{q}\left(\mathfrak{s l}_{2}\right)$. Inspired by their work, we investigate in this paper if there is any connection between the quantum system considered in Ref. 1 and representations of $U_{q}\left(\mathfrak{s l}_{2}\right)$. We shall show that, for a vector potential determined by the Weierstrass Zeta function, special elements of the unitary groups generated by the components of the physical momentum operator induce representations of $U_{q}\left(\mathfrak{s l}_{2}\right)$ on the Hilbert space $L^{2}\left(\mathbf{R}^{2}\right)$. This establishes a connection of a classical special function to $U_{q}\left(\mathfrak{s l}_{2}\right)$. A particular feature of the representations of $U_{q}\left(\mathfrak{s l}_{2}\right)$ given in this paper is that they have no finite-dimensional irreducible components.

The present paper is organized as follows. In Sec. II we consider a quantum system of a charged particle in $\mathbf{R}^{2}$ under the influence of a perpendicular magnetic field that may be singular at points in an infinite lattice [see (2.1)]. This is an extension of the framework of Ref. 1 to the case where the number of possible singular points of the magnetic field is infinite. Fundamental results in Ref. 1 continue to hold in the present case too with no significant modifications. As in the case of Ref. 1, the position operator and the physical momentum operator $\mathbf{P}=\left(P_{1}, P_{2}\right)$ of the particle give a representation of the CCR with two degrees of freedom if the magnetic field is concentrated on the infinite lattice. Spectral properties of the unitary operators generated by $P_{1}$ and $P_{2}$ are analyzed. Also, we show that, under some conditions for the vector potential of the magnetic field, $P_{1}$ and $P_{2}$ have permutation-reflection symmetries.

In Secs. III-V, we consider the special case where the vector potential $A=A_{1} d x+A_{2} d y$ (a 1-form) of the magnetic field is given by the Weierstrass Zeta function $\zeta$ in such a way that $\zeta=A_{2}+i A_{1}$ [see (3.2)-(3.4)]. We first show in Sec. III that, if the charge of the particle is not an integer, then special elements of the unitary groups generated by $P_{1}$ and $P_{2}$ give representations of the quantum plane. Unitary equivalences of these representations are discussed. As is shown in Ref. 8, a representation of the quantum plane with some additional properties induces representations of $U_{q}\left(\mathfrak{s l}_{2}\right)$. Applying this idea, we construct in Secs. IV and V representations of $U_{q}\left(\mathfrak{s l}_{2}\right)$ on $L^{2}\left(\mathbf{R}^{2}\right)$ and investigate some properties of them.

\section{A CHARGED PARTICLE IN A MAGNETIC FIELD WITH POSSIBLE SINGULARITIES ON AN INFINITE LATTICE}

\section{A. Representation of CCR}

We consider a quantum system of a charged particle with charge $\alpha \in \mathbf{R} \backslash\{0\}$ moving in the plane $\mathbf{R}^{2}=\{\mathbf{r}=(x, y) \mid x, y \in \mathbf{R}\}$ under the influence of a perpendicular magnetic field $B$ that may be singular at points in the infinite lattice,

$$
\mathbf{Z}_{\omega_{1}, \omega_{2}}^{2}:=\left\{\boldsymbol{\Omega}_{m, n}=\left(\omega_{1} m, \omega_{2} n\right) \mid m, n \in \mathbf{Z}\right\}, \neg
$$

where $\omega_{j}, j=1,2$, are positive constants and $\mathbf{Z}$ is the set of integers. A vector potential of the magnetic field $B$ is given by a continuously differentiable real 1-form $A=A_{1} d x+A_{2} d y$ on the nonsimply connected domain

$$
\left.\mathbf{M}=\mathbf{R}^{2} \backslash \mathbf{Z}_{\omega_{1}, \omega_{2}}^{2},\right\urcorner
$$

such that

$$
\left.B=D_{x} A_{2}-D_{y} A_{1},\right\urcorner
$$


in the distribution sense, where $D_{x}$ and $D_{y}$ are the generalized partial differential operators in the variables $x$ and $y$, respectively. We say that $A$ is flat on $\mathbf{M}$ if $B=0$ on $\mathbf{M}$, i.e., $B$ is concentrated on $\mathbf{Z}_{\omega_{1}, \omega_{2}}^{2}$ (in the sense of distribution).

Throughout this paper we use a physical unit system such that $\hbar$ (the Planck constant divided by $2 \pi)=c$ (the light velocity) $=1$. Let $\left(Q_{1}, Q_{2}\right)$ be the position operator of the particle, i.e., $Q_{1}$ and $Q_{2}$ are the maximal multiplication operators by $x$ and $y$ acting in the Hilbert space $L^{2}(\mathbf{M}) \cong L^{2}\left(\mathbf{R}^{2}\right)$, respectively. The physical (kinetic) momentum operator $\mathbf{P}=\left(P_{1}, P_{2}\right)$ of the particle is defined by the operators

$$
\left.\left.P_{j}=p_{j}-\alpha A_{j},\right\urcorner j=1,2,\right\urcorner
$$

acting in $L^{2}\left(\mathbf{R}^{2}\right)$, where $\left(p_{1}, p_{2}\right)$ is the momentum operator of the free particle:

$$
\left.p_{1}=-i D_{x},\right\urcorner p_{2}=-i D_{y} \cdot \neg
$$

We denote by $C_{0}^{m}(\mathbf{M})(m=0,1,2, \ldots)$ the space of $m$ times continuously differentiable functions on $\mathbf{M}$ with bounded support in $\mathbf{M}$. In the same way as in Ref. 1, we can prove the following fact.

Lemma 2.1:

(i) Each $P_{j}$ is essentially self-adjoint on $C_{0}^{l}(\mathbf{M})$. (We denote the closure of $P_{j}$ by the same symbol.)

(ii) Suppose that $A$ is flat on $\mathbf{M}$. Then $\left\{L^{2}\left(\mathbf{R}^{2}\right), C_{0}^{2}(\mathbf{M}),\left\{Q_{j}, P_{j}\right\}_{j=1}^{2}\right\}$ is a representation of the CCR with two degrees of freedom.

The analysis of the representation $\left\{L^{2}\left(\mathbf{R}^{2}\right), C_{0}^{2}(\mathbf{M}),\left\{Q_{j}, P_{j}\right\}_{j=1}^{2}\right\}$ in Lemma 2.1 can be done in quite the same way as in the case where $\mathbf{Z}_{\omega_{1}, \omega_{2}}^{2}$ is replaced by a finite discrete set in $\mathbf{R}^{2}$ (see Refs. 1,4 , and 6). Hence, as for that, we describe only results needed later. Let

$$
\left.\mathbf{S}_{1}=\mathbf{R} \backslash\left\{m \omega_{1}\right\}_{m \in \mathbf{Z}},\right\urcorner \quad \mathbf{S}_{2}=\mathbf{R} \backslash\left\{n \omega_{2}\right\}_{n \in \mathbf{Z}} .
$$

For $(x, y) \in \mathbf{R} \times \mathbf{S}_{2}$, we can define

$$
U_{1}(x, y)=\exp \left(-i \alpha \int_{0}^{x} A_{1}\left(x^{\prime}, y\right) d x^{\prime}\right) . \neg
$$

Then $U_{1}$ defines a unique unitary operator as a multiplication operator on $L^{2}\left(\mathbf{R}^{2}\right)$. Similarly, the function

$$
\left.\left.U_{2}(x, y)=\exp \left(-i \alpha \int_{0}^{y} A_{2}\left(x, y^{\prime}\right) d y^{\prime}\right),\right\urcorner(x, y) \in \mathbf{S}_{1} \times \mathbf{R},\right\urcorner
$$

defines a unique unitary operator as a multiplication operator on $L^{2}\left(\mathbf{R}^{2}\right)$. We then have operator equalities,

$$
\left.\left.P_{j}=U_{j}^{-1} p_{j} U_{j},\right\urcorner j=1,2 \cdot\right\urcorner
$$

It follows from these relations that, for all $t \in \mathbf{R}, \psi \in L^{2}(\mathbf{R})$, and for almost everywhere (a.e.) $(x, y)$,

$$
\left(e^{i t P_{1}} \psi\right)(x, y)=\exp \left(-i \alpha \int_{x}^{x+t} A_{1}\left(x^{\prime}, y\right) d x^{\prime}\right) \psi(x+t, y), \neg
$$




$$
\left(e^{i t P_{2}} \psi\right)(x, y)=\exp \left(-i \alpha \int_{y}^{y+t} A_{2}\left(x, y^{\prime}\right) d y^{\prime}\right) \psi(x, y+t) . \neg
$$

Let $C_{ \pm}(x, y ; s, t)(x, y, s, t \in \mathbf{R})$ be hook-shaped paths from $(x, y)$ to $(x+s, y+t)$ given by

$$
\begin{aligned}
& C_{-}(x, y ; s, t)=\{(x+\theta s, y) \mid 0 \leqslant \theta \leqslant 1\} \cup\{(x+s, y+\theta t) \mid 0 \leqslant \theta \leqslant 1\}, \\
& C_{+}(x, y ; s, t)=\{(x, y+\theta t) \mid 0 \leqslant \theta \leqslant 1\} \cup\{(x+\theta s, y+t) \mid 0 \leqslant \theta \leqslant 1\},
\end{aligned}
$$

and set

$$
\left.C(x, y ; s, t)=C_{+}(x, y ; s, t)^{-1} \circ C_{-}(x, y ; s, t),\right\urcorner
$$

the rectangular path: $(x, y) \rightarrow(x+s, y) \rightarrow(x+s, y+t) \rightarrow(x, y+t) \rightarrow(x, y)$. For $s, t \in \mathbf{R}$, we set

$$
\mathbf{S}_{1}^{(s)}=\mathbf{R} \backslash\left\{\omega_{1} m, \omega_{1} m-s \mid m \in \mathbf{Z}\right\}, \quad \mathbf{S}_{2}^{(t)}=\mathbf{R} \backslash\left\{\omega_{2} n, \omega_{2} n-t \mid n \in \mathbf{Z}\right\}, \neg
$$

and define

$$
\left.\left.\mathbf{M}_{s, t}=\mathbf{S}_{1}^{(s)} \times \mathbf{S}_{2}^{(t)},\right\urcorner \quad s, t \in \mathbf{R} .\right\urcorner
$$

For each $s, t \in \mathbf{R}$, we can define a function $\Phi_{s, t}^{A}$ on $\mathbf{M}_{s, t}$ by

$$
\left.\left.\Phi_{s, t}^{A}(x, y)=\int_{C(x, y ; s, t)} A,\right\urcorner \quad(x, y) \in \mathbf{M}_{s, t},\right\urcorner
$$

which physically means the magnetic flux passing through the interior domain of the closed curve $C(x, y ; s, t)$. Since $\mathbf{R}^{2} \backslash \mathbf{M}_{s, t}$ is a null set with respect to the two-dimensional Lebesgue measure, one can regard $\Phi_{s, t}^{A}$ as a real-valued function on $\mathbf{R}^{2}$, which is a.e. finite. Hence $\Phi_{s, t}^{A}$ defines a unique self-adjoint multiplication operator on $L^{2}\left(\mathbf{R}^{2}\right)$. We denote this operator by the same symbol. The following theorem gives commutation relations for the one parameter unitary groups generated by $Q_{j}$ and $P_{j}, j=1,2$.

Theorem 2.2. (cf. Theorem 2.1 in Ref. 1): For all $s, t \in \mathbf{R}$,

$$
\begin{gathered}
e^{i s Q_{j}} e^{i t P_{k}}=e^{-i s t \delta_{j k}} e^{i t P_{k}} e^{i s Q_{j}}, \neg \\
e^{i s P_{1}} e^{i t P_{2}}=e^{-i \alpha \Phi_{s, t}^{A}} e^{i t P_{2}} e^{i s P_{1}} . \neg
\end{gathered}
$$

Following Ref. 1, we say that the magnetic flux is locally quantized if, for all $s, t \in \mathbf{R}, \Phi_{s, t}^{A}$ is a $2 \pi \mathbf{Z} / \alpha$-valued function on $\mathbf{M}_{s, t}$.

Theorem 2.2 implies the following characterization of the representation $\left\{Q_{j}, P_{j}\right\}_{j=1}^{2}$ of CCR in the case where $A$ is flat [Lemma 2.1(ii)].

Theorem 2.3: Suppose that $A$ is flat on M. Then the representation $\left\{Q_{j}, P_{j}\right\}_{j=1}^{2}$ of $C C R$ is equivalent if and only if the magnetic flux is locally quantized.

Remark: (i) In the case where the magnetic flux is not locally quantized, formula (2.16) may be regarded as a mathematical form of the Aharonov-Bohm effect. ${ }^{9}$ Thus the inequivalent representation of $\left\{Q_{j}, P_{j}\right\}_{j=1}^{2}$ corresponds to the Aharonov-Bohm effect.

(ii) In operator theory, two self-adjoint operators, $S$ and $T$, on a Hilbert space are said to be strongly commuting if their spectral measures commute. It is well known that $S$ and $T$ are strongly commuting if and only if $e^{i a S} e^{i b T}=e^{i b T} e^{i a S}$ for all $a, b \in \mathbf{R}$ (Theorem VIII.13 in Ref. 10). It follows from this fact and (2.16) that $P_{1}$ and $P_{2}$ are strongly commuting if and only if the magnetic flux is locally quantized. 
Let $\omega_{0}>0$ be a number such that, for all $(m, n),\left(m^{\prime}, n^{\prime}\right) \in \mathbf{Z}^{2}$ with $(m, n) \neq\left(m^{\prime}, n^{\prime}\right)$, $\left\{\mathbf{r}|| \mathbf{r}-\boldsymbol{\Omega}_{m, n} \mid<\omega_{0}\right\} \cap\left\{\mathbf{r}|| \mathbf{r}-\boldsymbol{\Omega}_{m^{\prime}, n^{\prime}} \mid<\omega_{0}\right\}=\emptyset$. The following fact is easily proven.

Lemma 2.4: Suppose that $A$ is flat. Let $0<\delta<\omega_{0}$. Then

$$
\left.\gamma_{m, n}(A):=\int_{\left|\mathbf{r}-\mathbf{\Omega}_{m, n}\right|=\delta} A\right\urcorner
$$

is independent of $\delta$, where the orientation of the integral on the right-hand side (rhs) is taken to be anticlockwise. Moreover, for all $s, t \in \mathbf{R}$,

$$
\left.\Phi_{s, t}^{A}(x, y)=\boldsymbol{\epsilon}(s) \boldsymbol{\epsilon}(t) \sum_{\mathbf{\Omega}_{m, n} \in D(x, y ; s, t)} \gamma_{m, n}(A),\right\urcorner(x, y) \in \mathbf{M}_{s, t},
$$

where $D(x, y ; s, t)$ is the interior domain of $C(x, y ; s, t)$ and $\epsilon(t)$ is the sign function: $\epsilon(t)=1$ for $t \geqslant 0$; $\epsilon(t)=-1$ for $t<0$.

Theorem 2.3 and Lemma 2.4 imply the following fact.

Theorem 2.5: Suppose that $A$ is flat. Then the representation $\left\{Q_{j}, P_{j}\right\}_{j=1}^{2}$ of CCR is equivalent if and only if $\gamma_{m, n}(A) \in 2 \pi \mathbf{Z} / \alpha$ for all $m, n \in \mathbf{Z}$.

\section{B. Spectral properties}

For later use, we investigate spectral properties of some unitary operators. For a densely defined closed linear operator $T$ on a Hilbert space, we denote by $\sigma(T)\left[\operatorname{resp} ., \sigma_{p}(T)\right]$ the spectrum (resp., point spectrum) of $T$. We set

$$
\mathbb{T}=\{z \in \mathbf{C}|| z \mid=1\} \cdot \neg
$$

Lemma 2.6:

(i) $\sigma\left(P_{j}\right)=\mathbf{R}, \sigma_{p}\left(P_{j}\right)=\emptyset, j=1,2$.

(ii) For all $t \in \mathbf{R} \backslash\{0\}$ and $j=1,2, \sigma\left(e^{i t P_{j}}\right)=\mathbb{T}, \sigma_{p}\left(e^{i t P_{j}}\right)=\emptyset$.

Proof: (i) By (2.8), we have $\sigma\left(P_{j}\right)=\sigma\left(p_{j}\right)=\mathbf{R}, \sigma_{p}\left(P_{j}\right)=\sigma_{p}\left(p_{j}\right)=\emptyset$. (ii) This follows from part (i) and the spectral mapping theorem. $\urcorner$

Lemma 2.7: For all $s, t \in \mathbf{R} \backslash\{0\}$,

$$
\left.\sigma_{p}\left(e^{i s P_{1}} e^{i t P_{2}}\right)=\emptyset, \quad \sigma_{p}\left(e^{i t P_{2}} e^{i s P_{1}}\right)=\emptyset .\right\urcorner
$$

Proof: It follows from (2.9) and (2.10) that, for all $s, t \in \mathbf{R}$,

$$
\begin{aligned}
& \left.e^{i s P_{1}} e^{i t P_{2}}=\exp \left(-i \alpha \int_{C_{-}(x, y ; s, t)} A\right) e^{i s p_{1}} e^{i t p_{2}},\right\urcorner \\
& \left.e^{i t P_{2}} e^{i s P_{1}}=\exp \left(-i \alpha \int_{C_{+}(x, y ; s, t)} A\right) e^{i t p_{2}} e^{i s p_{1}} \cdot\right\urcorner
\end{aligned}
$$

Since $e^{i s P_{1}} e^{i t P_{2}}$ is unitary, we have $\sigma_{p}\left(e^{i s P_{1}} e^{i t P_{2}}\right) \subset \mathbb{T}$. Suppose that there exists a vector $\psi \in L^{2}\left(\mathbf{R}^{2}\right)$ and a constant $\lambda \in \mathbb{T}$ such that $e^{i s P_{1}} e^{i t P_{2}} \psi=\lambda \psi$. Then, by (2.20), we have $\exp \left(e^{-i \alpha} \int_{C_{-}(x, y ; s, t)} A\right) \psi(x+s, y+t)=\lambda \psi(x, y)$ a.e. $(x, y)$. Hence $|\psi(x, y)|=|\psi(x+s, y+t)|$ a.e. $(x, y)$, which, together with the fact $\psi \in L^{2}\left(\mathbf{R}^{2}\right)$, implies $\psi=0$. Thus the first formula of (2.19) follows. Similarly, using (2.21), we can prove the second one of (2.19). ᄀ

Proposition 2.8: Consider the case where the magnetic flux is locally quantized. Then, for all $t, s \in \mathbf{R} \backslash\{0\}$,

$$
\sigma\left(e^{i s P_{1}} e^{i t P_{2}}\right)=\sigma\left(e^{i t P_{1}} e^{i s P_{2}}\right)=\mathrm{T} .
$$


Proof: Under the present assumption, $P_{1}$ and $P_{2}$ strongly commute [see Remark (ii) after Theorem 2.3]. Hence, by the two variable functional calculus, we see that $s P_{1}+t P_{2}$ is essentially self-adjoint and $e^{i s P_{1}} e^{i t P_{2}}=e^{i t P_{2}} e^{i s P_{1}}=e^{i\left(\overline{s P_{1}+t P_{2}}\right)}$, where $\overline{s P_{1}+t P_{2}}$ is the closure of $s P_{1}+t P_{2}$. By the two variable functional calculus and Lemma 2.6(i), we have $\sigma\left(\overline{s P_{1}+t P_{2}}\right)=\mathbf{R}$. Thus, by the spectral mapping theorem, we obtain the desired result. $\neg$

Remark: We have been unable to identify $\sigma\left(e^{i s P_{1}} e^{i t P_{2}}\right)$ and $\sigma\left(e^{i t P_{2}} e^{i s P_{1}}\right)$ in the case where the magnetic field is not locally quantized. It would be interesting to see if there appears any difference from the case where the magnetic field is locally quantized. In the case of Dirac-Weyl operators defined in terms of $P_{1}$ and $P_{2}$, such differences exist; see Ref. 5. We leave this problem as an open problem.

\section{Permutation-reflection symmetry of the physical momentum operator}

Let $R_{j}: L^{2}\left(\mathbf{R}^{2}\right) \rightarrow L^{2}\left(\mathbf{R}^{2}\right), j=1,2$, be the unitary operators defined by

$$
\left.\left(R_{1} \psi\right)(x, y)=\psi(-y, x), \quad\left(R_{2} \psi\right)(x, y)=\psi(y,-x), \quad \psi \in L^{2}\left(\mathbf{R}^{2}\right) .\right\urcorner
$$

Then it is easy to see that

$$
\begin{gathered}
\left.R_{1} R_{2}=R_{2} R_{1}=I,\right\urcorner \\
\left.\left.R_{1} p_{1} R_{1}^{-1}=-p_{2},\right\urcorner R_{1} p_{2} R_{1}^{-1}=p_{1},\right\urcorner \\
\left.\left.R_{2} p_{1} R_{2}^{-1}=p_{2},\right\urcorner R_{2} p_{2} R_{2}^{-1}=-p_{1} \cdot\right\urcorner
\end{gathered}
$$

As usual, we denote by $z=x+i y$ the point in the complex plane $\mathbf{C}$ corresponding to $\mathbf{r}=(x, y) \in \mathbf{R}^{2}$. We set

$$
\left.\widetilde{A}(z)=A_{2}(x, y)+i A_{1}(x, y) \cdot\right\urcorner
$$

Proposition 2.9:

(i) Suppose that

$$
\widetilde{A}(i z)=-i \widetilde{A}(z) \cdot \neg
$$

Then

$$
\left.\left.R_{1} P_{1} R_{1}^{-1}=-P_{2},\right\urcorner R_{1} P_{2} R_{1}^{-1}=P_{1} \cdot\right\urcorner
$$

(ii) Suppose that

$$
\widetilde{A}(i z)=i \widetilde{A}(-z) \cdot \neg
$$

Then

$$
\left.\left.R_{2} P_{1} R_{2}^{-1}=P_{2},\right\urcorner \quad R_{2} P_{2} R_{2}^{-1}=-P_{1},\right\urcorner
$$

Proof: (i) Condition (2.27) is equivalent to that

$$
\left.A_{2}(-y, x)=A_{1}(x, y),\right\urcorner A_{1}(-y, x)=-A_{2}(x, y) .
$$

Hence, for all $\psi \in C_{0}^{1}(\mathbf{M})$,

$$
\left.\left.R_{1} P_{1} R_{1}^{-1} \psi=-P_{2} \psi,\right\urcorner R_{1} P_{2} R_{1}^{-1} \psi=P_{1} \psi .\right\urcorner
$$


Since $R_{1}$ leaves $C_{0}^{1}(\mathbf{M})$ invariant bijectively and $P_{j}, j=1,2$, are essentially self-adjoint on $C_{0}^{1}(\mathbf{M})$ [Lemma 2.1(i)], the vector equations in (2.31) extend to operator equalities (2.28).

(ii) Similar to part (i). $\urcorner$

In Sec. III, we shall consider the case of a vector potential satisfying (2.27) and (2.29).

\section{Small coupling limit}

Finally we consider the small coupling limit $\alpha \rightarrow 0$ of $P_{j}$.

Lemma 2.10: For all $t \in \mathbf{R}$ and $j=1,2$,

$$
\mathrm{s}-\lim _{\alpha \rightarrow 0} e^{i t P_{j}}=e^{i t p_{j}}, \neg
$$

where s-lim denotes strong limit.

Proof: For all $\psi \in C_{0}^{1}(\mathbf{M})$, we have $P_{j} \psi \rightarrow p_{j} \psi(\alpha \rightarrow 0)$. Note that $C_{0}^{1}(\mathbf{M})$ is a common core of $P_{j}$ and $p_{j}$. Hence, by general convergence theorems [Theorem VIII.25 (a) and Theorem VIII.21 in Ref. 10], we obtain (2.32).

\section{A VECTOR POTENTIAL GIVEN BY THE WEIERSTRASS ZETA FUNCTION AND REPRESENTATIONS OF THE QUANTUM PLANE}

We now specialize the vector potential $A$. We set

$$
\left.\left.\Omega_{m, n}=m \omega_{1}+i n \omega_{2},\right\urcorner \quad m, n \in \mathbf{Z} .\right\urcorner
$$

Let $\zeta(z)(z \in \mathbf{C})$ be the Weierstrass Zeta function with poles at $z=\Omega_{m, n}, m, n \in \mathbf{Z}$ :

$$
\left.\zeta(z)=\frac{1}{z}+\sum_{(m, n) \in \mathbf{Z}^{2} \backslash\{(0,0)\}}\left(\frac{1}{z-\Omega_{m, n}}+\frac{1}{\Omega_{m, n}}+\frac{z}{\Omega_{m, n}^{2\urcorner}}\right) \cdot\right\urcorner
$$

In what follows, we assume that the vector potential $A$ is given by $A=A_{1} d x+A_{2} d y$ with

$$
A_{1}(\mathbf{r})=\operatorname{Im} \zeta(z), \quad A_{2}(\mathbf{r})=\operatorname{Re} \zeta(z),
$$

so that

$$
\zeta(z)=A_{2}(\mathbf{r})+i A_{1}(\mathbf{r}) \cdot \neg
$$

Then, by the Cauchy-Riemann equation, $A$ is flat on $\mathbf{M}$.

In the present case, the constant $\gamma_{m, n}(A)$ defined by (2.17) is computed as

$$
\gamma_{m, n}(A)=2 \pi,
$$

independently of $(m, n) \in \mathbf{Z}^{2}$. Hence the magnetic flux is locally quantized if and only if $\alpha$ is an integer. Thus the local quantization of the magnetic flux is equivalent to the "charge quantization." The representation $\left\{Q_{j}, P_{j}\right\}_{j=1}^{2}$ of CCR in the present case is an inequivalent representation if and only if $\alpha$ is not an integer.

Let

$$
\left.q_{\alpha}=e^{2 \pi i \alpha} \cdot\right\urcorner
$$

Lemma 3.1: For all $m, n \in \mathbf{Z}$,

$$
e^{i n \omega_{2} P_{2}} e^{i m \omega_{1} P_{1}}=q_{\alpha}^{n m} e^{i m \omega_{1} P_{1}} e^{i n \omega_{2} P_{2}} \cdot \neg
$$


Proof: For all $(x, y) \in \mathbf{M}_{\omega_{1}, \omega_{2}}, D\left(x, y ; \omega_{1}, \omega_{2}\right)$ contains only one point in the lattice $\mathbf{Z}_{\omega_{1}, \omega_{2}}^{2}$. Hence, by Lemma 2.4 and (3.5), $\Phi_{\omega_{1}, \omega_{2}}^{A}=2 \pi$, a.e., which, together with (2.16), implies (3.7) with $m=n=1$. Using this relation repeatedly, we obtain (3.7) with $m, n \in \mathbf{N} \cup\{0\}$, whose adjoint gives (3.7) with $m, n<0$. Noting that $e^{i t P_{j}}(t \in \mathbf{R})$ is invertible with $\left(e^{i t P_{j}}\right)^{-1}=e^{-i t P_{j}}$, we obtain (3.7) with $m \geqslant 0, n<0$ or $m \leqslant 0, n>0$. ᄀ

Relation (3.7) naturally leads us to the quantum plane, ${ }^{11}$ which is defined to be the algebra generated by two elements $X, Y$ subject to the relation

$$
q X Y=Y X,
$$

with $q$ a parameter. ${ }^{12}$ We denote the quantum plane by $\mathbf{C}_{q}^{2}$.

For an algebra $\mathfrak{U}$, a set $(\pi, V)$ of a complex vector space $V$ and an algebraic homomorphism $\pi: \mathfrak{u} \rightarrow \operatorname{End}(V)$ is called a representation of $\mathfrak{U}$.

We denote by $\mathfrak{B}\left(L^{2}\left(\mathbf{R}^{2}\right)\right)$ the $*$ algebra of bounded linear operators on $L^{2}\left(\mathbf{R}^{2}\right)$. The following theorem immediately follows from Lemma 3.1.

Theorem 3.2: The following correspondence $\pi_{\alpha}:\{X, Y\} \rightarrow \mathfrak{B}\left(L^{2}\left(\mathbf{R}^{2}\right)\right)$ defines a representation of $\mathbf{C}_{q_{\alpha}}^{2}$ on $L^{2}\left(\mathbf{R}^{2}\right)$ :

$$
\left.\pi_{\alpha}(X)=e^{i \omega_{1} P_{1}}, \quad \pi_{\alpha}(Y)=e^{i \omega_{2} P_{2}}\right\urcorner
$$

It should be noted that, in the representation $\left(\pi_{\alpha}, L^{2}\left(\mathbf{R}^{2}\right)\right), \pi_{\alpha}(X)$ and $\pi_{\alpha}(Y)$ are unitary operators. Also, $q_{\alpha} \neq 1$ if and only if $\alpha \notin \mathbf{Z}$.

We have

$$
\left.\lim _{\alpha \rightarrow 0} q_{\alpha}=1,\right\urcorner
$$

which means that the small coupling limit $\alpha \rightarrow 0$ corresponds to the "classical limit" of deformation by the parameter $q_{\alpha}$.

The representation $\left(\pi_{\alpha}, L^{2}\left(\mathbf{R}^{2}\right)\right)$ has a nontrivial classical limit.

Proposition 3.3:

$$
\left.\mathrm{s}-\lim _{\alpha \rightarrow 0} \pi_{\alpha}(X)=e^{i \omega_{1} p_{1}} \neq I,\right\urcorner \quad \mathrm{s}-\lim _{\alpha \rightarrow 0} \pi_{\alpha}(Y)=e^{i \omega_{2} p_{2}} \neq I,
$$

where I denotes the identity operator on $L^{2}\left(\mathbf{R}^{2}\right)$.

Proof: This follows from Lemma 2.10.ᄀ

For a subalgebra $\mathfrak{M}$ of $\mathfrak{B}\left(L^{2}\left(\mathbf{R}^{2}\right)\right)$, we denote by $\mathfrak{M}^{\prime}$ the commutant of $\mathfrak{M}$ :

$$
\left.\mathfrak{M}^{\prime}=\left\{T \in \mathfrak{B}\left(L^{2}\left(\mathbf{R}^{2}\right)\right) \mid T S=S T, S \in \mathfrak{M}\right\} .\right\urcorner
$$

Let $\mathfrak{E}_{\alpha}$ be the algebra generated by $\left\{e^{i \omega_{1} P_{1}}, e^{i \omega_{2} P_{2}}\right\}$ :

$$
\mathfrak{E}_{\alpha}=\pi_{\alpha}\left(\mathbf{C}_{q}^{2} \alpha\right) \text {. }
$$

Lemma 3.4: $e^{ \pm 2 \pi i Q_{1} / \omega_{1}}, e^{ \pm 2 \pi i Q_{2} / \omega_{2}} \in \mathfrak{E}_{\alpha}^{\prime}$.

Proof: This follows from (2.15). $\neg$

By Lemma 3.1, we have

$$
\begin{gathered}
\left.q_{\alpha} e^{-i \omega_{2} P_{2}} e^{i \omega_{1} P_{1}}=e^{i \omega_{1} P_{1}} e^{-i \omega_{2} P_{2}}, \neg q_{\alpha} e^{i \omega_{2} P_{2}} e^{-i \omega_{1} P_{1}}=e^{-i \omega_{1} P_{1}} e^{i \omega_{2} P_{2}},\right\urcorner \\
\left.q_{\alpha} e^{-i \omega_{1} P_{1}} e^{-i \omega_{2} P_{2}}=e^{-i \omega_{2} P_{2}} e^{-i \omega_{1} P_{1}} \cdot\right\urcorner
\end{gathered}
$$


Hence, each of $\left\{e^{-i \omega_{2} P_{2}}, e^{i \omega_{1} P_{1}}\right\},\left\{e^{i \omega_{2} P_{2}}, e^{-i \omega_{1} P_{1}}\right\}$, and $\left\{e^{-\omega_{1} P_{1}}, e^{-i \omega_{2} P_{2}}\right\}$ gives a representation of $\mathbf{C}_{q_{\alpha}}^{2}$. We denote these representations by $\left(\pi_{\alpha}^{(j)}, L^{2}\left(\mathbf{R}^{2}\right)\right), j=1,2,3$, respectively.

Proposition 3.5: Suppose that

$$
\left.\omega_{1}=\omega_{2} \cdot\right\urcorner
$$

Then, each representation $\left(\pi_{\alpha}^{(j)}, L^{2}\left(\mathbf{R}^{2}\right)\right)$ is unitarily equivalent to $\left(\pi_{\alpha}, L^{2}\left(\mathbf{R}^{2}\right)\right)$.

Proof: It is easy to check that, if (3.13) is satisfied, then (2.27) and (2.29) hold with $\widetilde{A}(z)$ replaced by $\zeta(z)$. Hence we have (2.28) and (2.30), which imply that, for all $t \in \mathbf{R}$,

$$
\left.\left.\left.R_{1} e^{i t P_{1}} R_{1}^{-1}=e^{-i t P_{2}},\right\urcorner R_{1} e^{i t P_{2}} R_{1}^{-1}=e^{i t P_{1}},\right\urcorner R_{2} e^{i t P_{1}} R_{2}^{-1}=e^{i t P_{2}},\right\urcorner R_{2} e^{i t P_{2}} R_{2}^{-1}=e^{i t P_{1}} .
$$

These relations give a unitary equivalence between $\left(\pi_{\alpha}, L^{2}\left(\mathbf{R}^{2}\right)\right)$ and $\left(\pi_{\alpha}^{(j)}, L^{2}\left(\mathbf{R}^{2}\right)\right)(j=1,2)$. Moreover, (3.14) implies that

$$
R_{1}^{2} e^{i t P_{1}} R_{1}^{-2}=e^{-i t P_{1}}, \neg R_{2}^{2} e^{i t P_{2}} R_{2}^{-2}=e^{-i t P_{2}} .
$$

It is easy to see that $R_{1}^{2}=R_{2}^{2}$. Thus, the unitary equivalence between $\left(\pi_{\alpha}, L^{2}\left(\mathbf{R}^{2}\right)\right)$ and $\left(\pi_{\alpha}^{(3)}, L^{2}\left(\mathbf{R}^{2}\right)\right)$ follows. $\urcorner$

Remark: (i) In the case $\omega_{1} \neq \omega_{2}$, we have been unable to clarify whether $\left(\pi_{\alpha}, L^{2}\left(\mathbf{R}^{2}\right)\right)$, $\left(\pi_{\alpha}^{(j)}, L^{2}\left(\mathbf{R}^{2}\right)\right), j=1,2,3$, are unitarily equivalent to each other or not.

(ii) Relation (2.15) implies that

$$
\left.q_{\alpha} e^{2 \pi i \alpha Q_{j} / \omega_{j}} e^{i \omega_{j} P} P_{j}=e^{i \omega_{j} P} e^{2 \pi i \alpha Q_{j} / \omega_{j}},\right\urcorner \quad j=1,2 .
$$

Hence, for each $j=1,2\left\{e^{2 \pi i \alpha Q_{j} / \omega_{j}}, e^{i \omega_{j} P_{j}}\right\}$ gives a representation of $\mathbf{C}_{q_{\alpha}}^{2}$. It is a problem to clarify whether these representations and the representations $\left(\pi_{\alpha}, L^{2}\left(\mathbf{R}^{2}\right)\right),\left(\pi_{\alpha}^{(j)}, L^{2}\left(\mathbf{R}^{2}\right)\right), j=1,2,3$, are unitarily equivalent to each other or not.

In this paper we concentrate our attention on the representation $\left(\pi_{\alpha}, L^{2}\left(\mathbf{R}^{2}\right)\right)$. The methods developed in what follows apply also to the other representations of the quantum plane.

(iii) Consider the case where $\alpha$ is a rational number: $\alpha=p / r$ with $p \in \mathbf{Z}$ and $r \in \mathbf{Z} \backslash\{0\}$. Then $q_{\alpha}^{r}=1$. It follows from (3.7) that, for all $m, n \in \mathbf{Z}$ with $m n=r, e^{i m \omega_{1} P_{1}}$ and $e^{i n \omega_{2} P_{2}}$ commute. But, if $\alpha$ is irrational, then $e^{i m \omega_{1} P_{1}}$ and $e^{i n \omega_{2} P_{2}}$ do not commute for all $m, n \in \mathbf{Z} \backslash\{0\}$.

\section{REPRESENTATION OF $\boldsymbol{U}_{q}\left(\mathfrak{s l}_{2}\right)(\mathrm{I})$}

For a complex number $q \in \mathbf{C} \backslash\{0,1,-1\}$, the quantum group $U_{q}\left(\mathfrak{s l}_{2}\right)$ is defined to be the algebra generated by four elements $E, F, K, K^{-1}$ subject to the following relations: ${ }^{13}$

$$
\begin{gathered}
K K^{-1}=K^{-1} K=1, \\
K E K^{-1}=q^{2} E, \neg K F K^{-1}=q^{-2} F, \\
{[E, F]=\frac{K-K^{-1}}{q-q^{-1}} .}
\end{gathered}
$$

The Casimir element $C$ of $U_{q}\left(\mathfrak{s l}_{2}\right)$ is defined by

$$
C=\frac{q K-2+q^{-1} K^{-1}}{\left(q-q^{-1}\right)^{2}}+F E \cdot \neg
$$

Given a representation $(\pi, V)$ of the quantum plane $\mathbf{C}_{q}^{2}$ such that $\pi(X)$ and $\pi(Y)$ are bijective, we can construct a representation of $U_{q}\left(\mathfrak{s l}_{2}\right)$. 
Lemma 4.1 (cf. Ref. 8): Let $(\pi, V)$ be as above and $a, b, a^{\prime}, b^{\prime} \in \mathbf{C}$ be constants satisfying

$$
\left.a b q=a^{\prime} b^{\prime} q^{-1}=-\frac{1}{\left(q-q^{-1}\right)^{2}} \cdot\right\urcorner
$$

Then the following correspondence $\Pi:\left\{E, F, K, K^{-}\right\} \rightarrow \operatorname{End}(V)$ defines a representation of $U_{q}\left(\mathfrak{s} \mathfrak{L}_{2}\right)$ :

$$
\begin{gathered}
\Pi(E)=\pi(X)\left(a \pi(X)+a^{\prime} \pi(X)^{-1}\right) \pi(Y)^{-1}, \\
\Pi(F)=\pi(Y)\left(b \pi(X)+b^{\prime} \pi(X)^{-1}\right) \pi(X)^{-1}, \\
\left.\Pi(K)=\pi(X)^{2},\right\urcorner \Pi\left(K^{-1}\right)=\pi(X)^{-2} .
\end{gathered}
$$

In this representation, we have

$$
\Pi(C)=a^{\prime} b+a b^{\prime}-\frac{2}{\left(q-q^{-1}\right)^{2}} \cdot \neg
$$

Proof: Direct computations. ᄀ

Remark: By (4.2), we can write $\Pi(C)$ as

$$
\Pi(C)=\left(a^{\prime}+a q\right)\left(b+b^{\prime} q^{-1}\right) .
$$

In the rest of the paper, we assume that $A_{j}, j=1,2$, are given by (3.3) and

$$
\left.\alpha \notin \frac{\mathbf{Z}}{2} \cdot\right\urcorner
$$

Hence $q_{\alpha}^{2} \neq 1$.

Let $a_{\alpha}, a_{\alpha}^{\prime}, b_{\alpha}, b_{\alpha}^{\prime}$ be constants satisfying

$$
\left.a_{\alpha} b_{\alpha} q_{\alpha}=a_{\alpha}^{\prime} b_{\alpha}^{\prime} q_{\alpha}^{-1}=-\frac{1}{\left(q_{\alpha}-q_{\alpha}^{-1}\right)^{2}}=\frac{1}{4 \sin ^{2} 2 \pi \alpha} .\right\urcorner
$$

By Lemma 4.1 and Theorem 3.2, we have the following theorem.

Theorem 4.2: The following correspondence $\Pi_{\alpha}:\left\{E, F, K, K^{-1}\right\} \rightarrow \mathfrak{B}\left(L^{2}\left(\mathbf{R}^{2}\right)\right)$ defines a representation of $U_{q_{\alpha}}\left(\mathfrak{s} \mathfrak{l}_{2}\right)$ on $L^{2}\left(\mathbf{R}^{2}\right)$ :

$$
\begin{gathered}
\Pi_{\alpha}(E)=e^{i \omega_{1} P_{1}}\left(a_{\alpha} e^{i \omega_{1} P_{1}}+a_{\alpha}^{\prime} e^{-i \omega_{1} P_{1}}\right) e^{-i \omega_{2} P_{2}}, \\
\Pi_{\alpha}(F)=e^{i \omega_{2} P_{2}}\left(b_{\alpha} e^{i \omega_{1} P_{1}}+b_{\alpha}^{\prime} e^{-i \omega_{1} P_{1}}\right) e^{-i \omega_{1} P_{1}}, \\
\left.\Pi_{\alpha}(K)=e^{2 i \omega_{1} P_{1}},\right\urcorner \Pi_{\alpha}\left(K^{-1}\right)=e^{-2 i \omega_{1} P_{1}} .
\end{gathered}
$$

We investigate basic properties of the representation $\left(\Pi_{\alpha}, L^{2}\left(\mathbf{R}^{2}\right)\right)$.

Theorem 4.3: The representation $\left(\Pi_{\alpha}, L^{2}\left(\mathbf{R}^{2}\right)\right)$ has no weight vectors. In particular, there exists no nonzero finite-dimensional subspace $W$ such that $\left(\Pi_{\alpha}, W\right)$ gives a representation of $U_{q_{\alpha}}\left(\mathfrak{s l}_{2}\right)$.

Proof: A weight vector of weight $\lambda \in \mathbf{C}$ in the representation $\left(\Pi_{\alpha}, L^{2}\left(\mathbf{R}^{2}\right)\right)$ is a nonzero vector $\psi$ satisfying $\Pi_{\alpha}(K) \psi=q_{\alpha}^{\lambda} \psi$. Hence $\psi$ is an eigenvector of $e^{2 i \omega_{1} P_{1}}$. But, by Lemma 2.6(ii), $e^{2 i \omega_{1} P_{1}}$ has no eigenvectors. The last assertion in Theorem 4.3 follows from the well-known fact that any nonzero finite-dimensional $U_{q}\left(\mathfrak{s L}_{2}\right)$-module contains a highest weight vector (Proposition VI.3.3 in Ref. 11). ᄀ 
Let

$$
\left.\mathfrak{U}_{\alpha}=\Pi_{\alpha}\left(U_{q_{\alpha}}\left(\mathfrak{s l}_{2}\right)\right) .\right\urcorner
$$

Theorem 4.4: Suppose that $a_{\alpha}^{\prime}=\bar{b}_{\alpha}, b_{\alpha}^{\prime}=\bar{a}_{\alpha}(\bar{z}$ denotes the complex conjugate of the complex number $z$ ). Then

(i) $\mathfrak{U}_{\alpha}$ is a * subalgebra of $\mathfrak{B}\left(L^{2}\left(\mathbf{R}^{2}\right)\right)$.

(ii) The representation $\left(\Pi_{\alpha}, L^{2}\left(\mathbf{R}^{2}\right)\right)$ is completely reducible.

Proof: (i) In the present assumption, we have

$$
\Pi_{\alpha}(E)^{*}=\Pi_{\alpha}(F)
$$

where $T^{*}$ denotes the adjoint of operator $T$. It is obvious that $\Pi_{\alpha}(K)^{*}=\Pi_{\alpha}\left(K^{-1}\right)$. Hence it follows that $\mathfrak{U}_{\alpha}$ is self-adjoint (i.e., $T \in \mathfrak{U}_{\alpha} \Rightarrow T^{*} \in \mathfrak{U}_{\alpha}$ ). Thus part (i) follows.

(ii) As in Lemma 3.4, we have

$$
\left\{e^{ \pm 2 \pi i Q_{j} / \omega_{j}}\right\}_{j=1}^{2} \subset \mathfrak{U}_{\alpha}^{\prime}, \neg
$$

which implies that $\mathfrak{U}_{\alpha}^{\prime} \neq \mathbf{C} I$. As proven in part (i), $\mathfrak{U}_{\alpha}$ is self-adjoint. Hence $\mathfrak{U}_{\alpha}$ is not irreducible (Proposition 2.3.8 in Ref. 14). Let $W$ be any closed subspace of $L^{2}\left(\mathbf{R}^{2}\right)$, which is invariant under the action of $\mathfrak{U}_{\alpha}$. Since $\mathfrak{U}_{\alpha}$ is self-adjoint, it follows that $W^{\perp}$ [the orthogonal complement of $W$ in $\left.L^{2}\left(\mathbf{R}^{2}\right)\right]$ is also invariant under the action of $\mathfrak{U}_{\alpha}$. Thus $\left(\Pi_{\alpha}, L^{2}\left(\mathbf{R}^{2}\right)\right)$ is completely reducible.

For a subset $\mathfrak{M}$ of $\mathfrak{B}\left(L^{2}\left(\mathbf{R}^{2}\right)\right)$, we denote by $\overline{\mathfrak{M}}$ the closure of $\mathfrak{M}$ with respect to the operator norm.

We denote by $\mathfrak{F}_{\alpha}$ the algebra generated by $e^{ \pm 2 i \omega_{1} P_{1}}, e^{ \pm i \omega_{2} P_{2}}$, which is a $*$ subalgebra of $\mathfrak{B}\left(L^{2}\left(\mathbf{R}^{2}\right)\right)$, so that $\frac{\mathfrak{F}_{\alpha}}{\mathfrak{F}_{\alpha}}$ is a $C^{*}$ subalgebra.

Theorem 4.5: Suppose that $\left|a_{\alpha}\right| \neq\left|a_{\alpha}^{\prime}\right|,\left|b_{\alpha}\right| \neq\left|b_{\alpha}^{\prime}\right|$. Then

(i)

$$
\left.\overline{\mathfrak{F}_{\alpha}}=\overline{\mathfrak{U}_{\alpha}} \cdot\right\urcorner
$$

(ii) $\overline{\mathfrak{U}_{\alpha}}$ is completely reducible.

Proof: (i) For simplicity, we set $a=a_{\alpha}, a^{\prime}=a_{\alpha}^{\prime}, b=b_{\alpha}, b^{\prime}=b_{\alpha}^{\prime}$. We have

$$
\left.\Pi_{\alpha}(E)=S e^{-i \omega_{2} P_{2}}, \neg \Pi_{\alpha}(F)=e^{i \omega_{2} P_{2}} T,\right\urcorner
$$

with

$$
\left.S=a e^{2 i \omega_{1} P_{1}}+a^{\prime},\right\urcorner T=b+b^{\prime} e^{-2 i \omega_{1} P_{1}} .
$$

Hence it follows that $\mathfrak{U}_{\alpha} \subset \mathfrak{F}_{\alpha}$, implying

$$
\left.\overline{\mathfrak{U}_{\alpha}} \subset \overline{\mathfrak{F}_{\alpha}} \cdot\right\urcorner
$$

To prove the converse inclusion relation, we express $e^{ \pm i \omega_{2} P_{2}}$ in terms of $\Pi_{\alpha}(E), \Pi_{\alpha}(F)$, $\Pi_{\alpha}(K)$, and $\Pi_{\alpha}\left(K^{-1}\right)$. We first consider the case $|a| /\left|a^{\prime}\right|<1$. We can write

$$
S=a \Pi_{\alpha}(K)+a^{\prime}=a^{\prime}\left(1+\frac{a}{a^{\prime}} \Pi_{\alpha}(K)\right) .
$$

We have $\left\|a \Pi_{\alpha}(K) / a^{\prime}\right\|=|a| /\left|a^{\prime}\right|<1$, where $\|L\|$ with operator $L \in \mathfrak{B}\left(L^{2}\left(\mathbf{R}^{2}\right)\right)$ denotes the operator norm of $L$. Hence, $S$ is bijective with 


$$
S^{-1}=\frac{1}{a^{\prime}} \sum_{n=0}^{\infty}(-1)^{n}\left(\frac{a}{a^{\prime}}\right)^{n} \Pi_{\alpha}(K)^{n}
$$

in the operator norm topology. Therefore we obtain

$$
e^{-i \omega_{2} P_{2}}=S^{-1} \Pi_{\alpha}(E) \in \overline{\mathfrak{U}_{\alpha}} .
$$

In the case $|a| /\left|a^{\prime}\right|>1$, we write

$$
S=a \Pi_{\alpha}(K)\left(1+\frac{a^{\prime}}{a} \Pi_{\alpha}\left(K^{-1}\right)\right) .
$$

Then, in the same way as in the preceding case, we can show that $S$ is bijective with

$$
S^{-1}=a^{-1} \sum_{n=0}^{\infty}(-1)^{n}\left(\frac{a^{\prime}}{a}\right)^{n} \Pi_{\alpha}\left(K^{-1}\right)^{n+1},
$$

in the operator norm topology. Hence $e^{-i \omega_{2} P_{2}} \in \overline{\mathfrak{U}_{\alpha}}$. Similarly, the second relation in (4.9) implies that $e^{i \omega_{2} P_{2}} \in \overline{\mathfrak{U}_{\alpha}}$. Thus, $\overline{\mathfrak{F}_{\alpha}} \subset \overline{\mathfrak{U}_{\alpha}}$, which, together with (4.10), gives (4.8).

(ii) Relation (4.7) implies that

$$
\left\{e^{ \pm 2 \pi i Q_{j} / \omega_{j}}\right\}_{j=1}^{2} \subset \overline{\mathfrak{U}_{\alpha}^{\prime}}
$$

By the preceding result, $\overline{\mathfrak{U}_{\alpha}}$ is self-adjoint (in fact, a $C^{*}$ subalgebra). Thus, by the same reasoning as in the proof of Theorem 4.4(ii), we obtain the desired result. $\urcorner$

Remark: In the case $a_{\alpha}= \pm a_{\alpha}^{\prime}, S$ is injective, but not surjective [Lemma 2.6(ii)]. Hence $S^{-1}$ is unbounded. The same applies to $T$ in the case $b_{\alpha}= \pm b_{\alpha}^{\prime}$.

For $(m, n) \in \mathbf{Z}^{2}$, we define a function $F_{m, n}(x, y)$ by

$$
F_{m, n}(x, y)=\left\{\begin{array}{l}
\exp \left(-i \alpha \int_{C_{-}\left(x, y ; 2 m \omega_{1}, n \omega_{2}\right)} A\right) ; \neg(x, y) \in \mathbf{M}_{\omega_{1}, \omega_{2}}, \\
0 ;\urcorner(x, y) \notin \mathbf{M}_{\omega_{1}, \omega_{2}} .
\end{array}\right.
$$

For $\psi \in L^{2}\left(\mathbf{R}^{2}\right)$, we set

$$
\left.\psi_{m, n}(x, y)=\psi\left(x+2 m \omega_{1}, y+n \omega_{2}\right), \quad(m, n) \in \mathbf{Z}^{2} .\right\urcorner
$$

Corollary 4.6: Suppose that $\left|a_{\alpha}\right| \neq\left|a_{\alpha}^{\prime}\right|,\left|b_{\alpha}\right| \neq\left|b_{\alpha}^{\prime}\right|$. Let $W$ be any irreducible closed subspace of the representation $\left(\Pi_{\alpha}, L^{2}\left(\mathbf{R}^{2}\right)\right)$. Then each nonzero vector $\psi \in W$ is cyclic and $W$ is generated by vectors of the form $F_{m, n} \psi_{m, n},(m, n) \in \mathbf{Z}^{2}$.

Proof: The first half is due to a general fact (Proposition 2.3.8 in Ref. 14). By Theorem 4.5(i), $W$ is generated by vectors of the form

$$
\phi_{m, n}=e^{2 i m \omega_{1} P_{1}} e^{i n \omega_{2} P_{2}} \psi, \neg(m, n) \in \mathbf{Z}^{2} .
$$

Using (2.20), we see that $\left.\phi_{m, n}=F_{m, n} \psi_{m, n}.\right\urcorner$

Corollary 4.6 clarifies the structure of any irreducible closed subspace $W$ of the representation $\left(\Pi_{\alpha}, L^{2}\left(\mathbf{R}^{2}\right)\right)$ in the case $\left|a_{\alpha}\right| \neq\left|a_{\alpha}^{\prime}\right|,\left|b_{\alpha}\right| \neq\left|b_{\alpha}^{\prime}\right|$. By Theorem 4.3, $\operatorname{dim} W=\infty$. 


\section{REPRESENTATION OF $\boldsymbol{U}_{q}\left(\mathfrak{s l}_{2}\right)$ (II)}

In this section, we construct representations of $U_{q}\left(\mathfrak{s l}_{2}\right)$ that are different from the one given in Sec. IV.

Lemma 5.1 (cf. Ref. 8): Suppose that $q \in \mathbf{C} \backslash\{0,1,-1\}$. Let $(\pi, V)$ be a representation of $\mathbf{C}_{q^{2}}^{2}$ [i.e., $\left.q^{2} \pi(X) \pi(Y)=\pi(Y) \pi(X)\right]$ with the following properties.

(i) $\pi(X)$ and $\pi(Y)$ are bijective on $V$.

(ii) There exists a bijection $Z \in \operatorname{End}(V)$ such that $Z^{2}=\pi(Y)^{-1} \pi(X)$.

Let $c_{ \pm}$and $d_{ \pm}$be constants satisfying

$$
c_{ \pm} d_{ \pm}= \pm \frac{1}{\left(q-q^{-1}\right)^{2}} \cdot \neg
$$

Then the following correspondences $\Pi_{ \pm}:\left\{E, F, K, K^{-1}\right\} \rightarrow$ End $(V)$ define representations of $U_{q}\left(\mathfrak{s l}_{2}\right)$ :

$$
\begin{gathered}
\Pi_{ \pm}(E)=c_{ \pm} Z\left(\pi(X)^{-1}+\pi(Y)^{-1}\right), \\
\Pi_{ \pm}(F)=d_{ \pm}(\pi(X)+\pi(Y)) Z^{-1}, \\
\Pi_{ \pm}(K)= \pm q^{-1} \pi(Y)^{-1} \pi(X), \\
\Pi_{ \pm}\left(K^{-1}\right)= \pm q \pi(X)^{-1} \pi(Y) .
\end{gathered}
$$

In these representations, we have

$$
\Pi_{ \pm}(C)= \pm \frac{\left(1+q^{2}\right) \pi(Y)^{-1} \pi(X)+\left(1+q^{-2}\right) \pi(X)^{-1} \pi(Y)}{\left(q-q^{-1}\right)^{2}} . \neg
$$

Proof: Direct computations.ᄀ

To apply Lemma 5.1 with the representation $\left(\pi_{\alpha}, L^{2}\left(\mathbf{R}^{2}\right)\right)$ of $\mathbf{C}_{q_{\alpha}}^{2}$ given in Theorem 3.2, we need the following lemma.

Lemma 5.2: Let $U$ be a unitary operator on a Hilbert space $\mathscr{H}$. Then there exists a unitary operator $T$ on $\mathscr{H}$ such that

$$
T^{2}=U \cdot \neg
$$

Proof: By the spectral theorem for unitary operators, there exists a unique resolution of identity $F(\theta)$ such that $F(0)=0, F(2 \pi)=I$, and $U=\int_{0}^{2 \pi} e^{i \theta} d F(\theta)$. Let $T=\int_{0}^{2 \pi} e^{i \theta / 2} d F(\theta)$. Then, by the functional calculus, $T$ is unitary and (5.3) holds. $\neg$

The operator $e^{-i \omega_{2} P_{2}} e^{i \omega_{1} P_{1}}$ is unitary on $L^{2}\left(\mathbf{R}^{2}\right)$. Hence, by Lemma 5.2, there exists a unitary operator $Z_{\alpha}$ on $L^{2}\left(\mathbf{R}^{2}\right)$ satisfying

$$
Z_{\alpha}^{2}=e^{-i \omega_{2} P_{2}} e^{i \omega_{1} P_{1}} \neg
$$

Let

$$
q_{\alpha}^{1 / 2}:=e^{i \pi \alpha} \cdot \neg
$$

Applying Lemma 5.1 with $\pi(X)=e^{i \omega_{1} P_{1}}, \pi(Y)=e^{i \omega_{2} P_{2}}$, we obtain the following theorem.

Theorem 5.3: Let $c_{ \pm}(\alpha)$ and $d_{ \pm}(\alpha)$ be constants satisfying

$$
\left.c_{ \pm}(\alpha) d_{ \pm}(\alpha)= \pm \frac{1}{\left(q_{\alpha}^{1 / 2}-q_{\alpha}^{-1 / 2}\right)^{2}}=\mp \frac{1}{4 \sin ^{2} \pi \alpha} .\right\urcorner
$$


Then the following correspondences $\Pi_{\alpha}^{ \pm}:\left\{E, F, K, K^{-1}\right\} \rightarrow \mathfrak{B}\left(L^{2}\left(\mathbf{R}^{2}\right)\right)$ define representations of $U_{q}\left(\mathfrak{s l}_{2}\right)$ with $q=q_{\alpha}^{1 / 2}$ :

$$
\begin{gathered}
\Pi_{\alpha}^{ \pm}(E)=c_{ \pm}(\alpha) Z_{\alpha}\left(e^{-i \omega_{1} P_{1}}+e^{-i \omega_{2} P_{2}}\right), \\
\Pi_{\alpha}^{ \pm}(F)=d_{ \pm}(\alpha)\left(e^{i \omega_{1} P_{1}}+e^{i \omega_{2} P_{2}}\right) Z_{\alpha}^{-1}, \\
\Pi_{\alpha}^{ \pm}(K)= \pm q_{\alpha}^{-1 / 2} e^{-i \omega_{2} P_{2}} e^{i \omega_{1} P_{1}}, \\
\Pi_{\alpha}^{ \pm}\left(K^{-1}\right)= \pm q_{\alpha}^{1 / 2} e^{-i \omega_{1} P_{1}} e^{i \omega_{2} P_{2}} .
\end{gathered}
$$

In these representations, we have

$$
\left.\Pi_{\alpha}^{ \pm}(C)= \pm \frac{\left(1+q_{\alpha}\right) e^{-i \omega_{2} P_{2}} e^{i \omega_{1} P_{1}}+\left(1+q_{\alpha}^{-1}\right) e^{-i \omega_{1} P_{1}} e^{i \omega_{2} P_{2}}}{\left(q_{\alpha}^{1 / 2}-q_{\alpha}^{-1 / 2}\right)^{2}} .\right\urcorner
$$

By Lemma 2.7, we obtain the following theorem.

Theorem 5.4: The representations $\left(\Pi_{\alpha}^{ \pm}, L^{2}\left(\mathbf{R}^{2}\right)\right)$ have no weight vectors. In particular, there exist no nonzero finite-dimensional subspaces $W_{ \pm}$, such that $\left(\Pi_{\alpha}^{ \pm}, W_{ \pm}\right)$give representations of

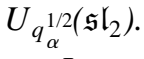

Let

$$
\left.\mathfrak{U}_{\alpha}^{ \pm}=\Pi_{\alpha}^{ \pm}\left(U_{q_{\alpha}^{1 / 2}}\left(\mathfrak{s l}_{2}\right)\right) \cdot\right\urcorner
$$

Theorem 5.5: Let $\overline{c_{ \pm}(\alpha)}=d_{ \pm}(\alpha)$. Then

(i) $\mathfrak{U}_{\alpha}^{ \pm}$are $*$ subalgebras of $\mathfrak{B}\left(L^{2}\left(\mathbf{R}^{2}\right)\right)$.

(ii) $\left(\Pi_{\alpha}^{ \pm}, L^{2}\left(\mathbf{R}^{2}\right)\right)$ are completely reducible.

Proof: Under the assumption, $\mathfrak{U}_{\alpha}^{ \pm}$are self-adjoint, since we have

$$
\Pi_{\alpha}^{ \pm}(E)^{*}=\Pi_{\alpha}^{ \pm}(F), \neg \Pi_{\alpha}^{ \pm}(K)^{*}=\Pi_{\alpha}^{ \pm}\left(K^{-1}\right) .
$$

Note that, if $T \in \mathfrak{B}\left(L^{2}\left(\mathbf{R}^{2}\right)\right)$ commutes with $e^{-i \omega_{2} P_{2}} e^{i \omega_{1} P_{1}}$, then $T$ commutes with $Z_{\alpha}$. In particular, $Z_{\alpha}$ commutes with $e^{2 \pi i Q_{j} / \omega_{j}}, j=1,2$. Hence $\left\{e^{2 \pi i Q_{j} / \omega_{j}}\right\}_{j=1}^{2} \subset \mathfrak{U}_{\alpha}^{ \pm \prime}$. Thus, by the same reasoning as in the proof of Theorem 4.4, we obtain the desired result. $\urcorner$

Note that $q_{2 \alpha}^{1 / 2}=q_{\alpha}$. Hence $\left(\Pi_{2 \alpha}^{ \pm}, L^{2}\left(\mathbf{R}^{2}\right)\right)$ are representations of $U_{q}\left(\mathfrak{s l}_{2}\right)$ with $q=q_{\alpha}$. It is natural to ask if these representations are equivalent to the representation $\left(\Pi_{\alpha}, L^{2}\left(\mathbf{R}^{2}\right)\right)$ constructed in the preceding section.

Theorem 5.6: The representations $\left(\Pi_{2 \alpha}^{ \pm} L^{2}\left(\mathbf{R}^{2}\right)\right)$ are not equivalent to $\left(\Pi_{\alpha}, L^{2}\left(\mathbf{R}^{2}\right)\right)$.

Proof: By (5.7), (2.20), and (2.21), we have, for all $\psi \in L^{2}\left(\mathbf{R}^{2}\right)$,

$$
\begin{aligned}
\left(\Pi_{2 \alpha}^{ \pm}(C) \psi\right)(x, y)= & \pm \frac{1+q_{\alpha}^{2}}{\left(q_{\alpha}-q_{\alpha}^{-1}\right)^{2}} \exp \left(-2 i \alpha \int_{C_{+}\left(x, y ; \omega_{1},-\omega_{2}\right)} A\right) \psi\left(x+\omega_{1}, y-\omega_{2}\right) \\
& \pm \frac{1+q_{\alpha}^{-2}}{\left(q_{\alpha}-q_{\alpha}^{-1}\right)^{2}} \exp \left(-2 i \alpha \int_{C_{-}\left(x, y ;-\omega_{1}, \omega_{2}\right)} A\right) \psi\left(x-\omega_{1}, y+\omega_{2}\right), \neg \text { a.e. }
\end{aligned}
$$

From this expression, it is seen that $\Pi_{2 \alpha}^{ \pm}(C)$ are not scalar multiples of the identity. [For example, consider a function $\psi \in L^{2}\left(\mathbf{R}^{2}\right), \psi \neq 0$, with support in $S=\left(0, \omega_{1}\right) \times\left(0, \omega_{2}\right)$. Then the support of $\Pi_{2 \alpha}^{ \pm}(C) \psi$ is outside of $S$.] On the other hand, by $(4.3), \Pi_{\alpha}(C)$ is a scalar multiple of the identity. Thus, the desired result follows. $\urcorner$ 
We can prove a more detailed fact on the inequivalence between $\left(\Pi_{2 \alpha}^{ \pm}, L^{2}\left(\mathbf{R}^{2}\right)\right)$ and $\left(\Pi_{\alpha}, L^{2}\left(\mathbf{R}^{2}\right)\right)$. For this purpose, we prepare a lemma. Let

$$
\left.h_{\alpha}^{ \pm}(\lambda)= \pm \frac{\left(1+q_{\alpha}\right) \lambda+\left(1+q_{\alpha}^{-1}\right) \bar{\lambda}}{\left(q_{\alpha}^{1 / 2}-q_{\alpha}^{-1 / 2}\right)^{2}}, \neg \quad \lambda \in \mathbf{C} .\right\urcorner
$$

Note that $h_{\alpha}^{ \pm}$are real valued.

Lemma 5.7:

(i)

$$
\left.\sigma\left(\Pi_{\alpha}^{ \pm}(C)\right)=\left\{h_{\alpha}^{ \pm}(\lambda) \mid \lambda \in \sigma\left(e^{-i \omega_{2} P_{2}} e^{i \omega_{1} P_{1}}\right)\right\} .\right\urcorner
$$

In particular,

$$
\left.\sigma\left(\Pi_{\alpha}^{ \pm}(C)\right) \subset\left[-R_{\alpha}, R_{\alpha}\right],\right\urcorner
$$

where

$$
\left.R_{\alpha}=\frac{2\left|1+q_{\alpha}\right|}{\left|q_{\alpha}^{1 / 2}-q_{\alpha}^{-1 / 2}\right|^{2}}=\frac{|\cos \pi \alpha|}{\sin ^{2} \pi \alpha} .\right\urcorner
$$

(ii)

$$
\sigma_{p}\left(\Pi_{\alpha}^{ \pm}(C)\right)=\emptyset . \neg
$$

Proof: (i) We first note that

$$
U_{\alpha}:=e^{-i \omega_{2} P_{2}} e^{i \omega_{1} P_{1}}
$$

is unitary and $U_{\alpha}^{-1}=e^{-i \omega_{1} P_{1}} e^{i \omega_{2} P_{2}}$. We have

$$
\Pi_{\alpha}^{ \pm}(C)= \pm \frac{\left(1+q_{\alpha}\right) U_{\alpha}+\left(1+q_{\alpha}^{-1}\right) U_{\alpha}^{-1}}{\left(q_{\alpha}^{1 / 2}-q_{\alpha}^{-1 / 2}\right)^{2}} .
$$

Relation (5.10) follows from this expression and the spectral mapping theorem for unitary operators. It is easy to see that there exists a constant $\delta_{\alpha} \in[0,2 \pi)$ such that

$$
h_{\alpha}^{ \pm}\left(e^{i \theta}\right)= \pm R_{\alpha} \cos \left(\theta+\delta_{\alpha}\right), \quad \theta \in \mathbf{R} .
$$

Hence (5.11) follows.

(ii) Suppose that $\sigma_{p}\left(\Pi_{\alpha}^{+}(C)\right) \neq$ and $\lambda \in \sigma_{p}\left(\Pi_{\alpha}^{+}(C)\right)$. Then, by (5.11), $-R_{\alpha} \leqslant \lambda \leqslant R_{\alpha}$ and there exists a nonzero vector $\psi \in L^{2}\left(\mathbf{R}^{2}\right)$ such that $\Pi_{\alpha}^{+}(C) \psi=\lambda \psi$. Let $E$ be the spectral measure of $U_{\alpha}$ :

$$
U_{\alpha}=\int_{0}^{2 \pi} e^{i \theta} d E(\theta)
$$

Then we have

$$
0=\left\|\Pi_{\alpha}^{+}(C) \psi-\lambda \psi\right\|^{2}=\int_{0}^{2 \pi}\left|h_{\alpha}^{+}\left(e^{i \theta}\right)-\lambda\right|^{2} d\|E(\theta) \psi\|^{2},
$$


which implies that the support of the measure $\|E(\cdot) \psi\|^{2}$ is included in the set $\left\{\theta \in[0,2 \pi] \mid h_{\alpha}^{+}\left(e^{i \theta}\right)=\lambda\right\}$. By (5.14), the equation $h_{\alpha}^{+}\left(e^{i \theta}\right)=\lambda$ has at most two solutions: $\theta_{j} \in[0,2 \pi), j=1,2$. This implies that one of $E\left(\left\{\theta_{j}\right\}\right) \psi(j=1,2)$ is not zero and an eigenvector of $U_{\alpha}$ with eigenvalue $e^{i \theta_{j}}$. But this contradicts Lemma 2.7. Thus $\sigma_{p}\left(\Pi_{\alpha}^{+}(C)\right)$ must be empty. Similarly, we can prove $\sigma_{p}\left(\Pi_{\alpha}^{-}(C)\right)=\emptyset$.

Theorem 5.8: Let $\left(\Pi_{\alpha}, W\right)$ be any irreducible component of $\left(\Pi_{\alpha}, L^{2}\left(\mathbf{R}^{2}\right)\right)\left(W \subset L^{2}\left(\mathbf{R}^{2}\right)\right)$. Then $\left(\Pi_{\alpha}, W\right)$ is not equivalent to any irreducible component of $\left(\Pi_{2 \alpha}^{ \pm} L^{2}\left(\mathbf{R}^{2}\right)\right)$.

Proof: By Lemma 5.7(ii), $\Pi_{2 \alpha}^{ \pm}(C)$ are not scalar multiples of the identity in any irreducible components of $\left(\Pi_{2 \alpha}^{+}, L^{2}\left(\mathbf{R}^{2}\right)\right)$. Thus, the desired result follows. $\neg$

Remark: It is an open problem to clarify whether $\left(\Pi_{\alpha}^{+}, L^{2}\left(\mathbf{R}^{2}\right)\right)$ is equivalent to $\left(\Pi_{\alpha}^{-}, L^{2}\left(\mathbf{R}^{2}\right)\right)$ or not.

\section{ACKNOWLEDGMENT}

This work was supported by the Grant-In-Aid No. 07640152 for science research from the Ministry of Education.

${ }^{1}$ A. Arai, "Momentum operators with gauge potentials, local quantization of magnetic flux, and representation of canonical commutation relations," J. Math. Phys. 33, 3374-3378 (1992).

${ }^{2}$ C. R. Putnam, Commutation Properties of Hilbert Space Operators (Springer-Verlag, Berlin, 1967).

${ }^{3}$ Y. Aharonov and D. Bohm, "Significance of electromagnetic potentials in the quantum theory," Phys. Rev. 115, 485-491 (1959).

${ }^{4}$ H. Kurose and H. Nakazato, "Geometric construction of *-representation of the Weyl algebra with degree 2," preprint, 1994.

${ }^{5}$ A. Arai, "Properties of the Dirac-Weyl operator with a strongly singular gauge potential," J. Math. Phys. 34, 915-935 (1993).

${ }^{6}$ A. Arai, "Gauge theory on a non-simply conneted domain and representations of canonical commutation relations," J. Math. Phys. 36, 2569-2580 (1995).

${ }^{7}$ A. Arai, "Representation of canonical commutation relations in a gauge theory, the Aharanov-Bohm effect, and the Dirac-Weyl operator,' J. Nonlin. Math. Phys. 2, 247-262 (1995).

${ }^{8}$ P. B. Wiegmann and A. V. Zabrodin, "Quantum groups and magnetic translations Bethe ansatz for Asbel-Hofstadter problem,” Nucl. Phys. B 422, 495-514 (1994).

${ }^{9}$ See Remark 2.3 in Ref. 5.

${ }^{10}$ M. Reed and B. Simon, Methods of Modern Mathematical Physics (Academic, New York, 1972), Vol. I.

${ }^{11}$ C. Kassel, Quantum Groups (Springer-Verlag, New York, 1995).

${ }^{12}$ In this paper, an algebra means an algebra over the complex field $\mathbf{C}$.

${ }^{13}$ See, e.g., Chap. VI, Definition VI.1.1 in Ref. 11.

${ }^{14}$ O. Bratteli and D. W. Robinson, Operator Algebra and Quantum Statistical Mechanics 1 (Springer-Verlag, New York, 1979). 